Classification

Physics Abstracts

$73.20 \mathrm{~F}-74.70 \mathrm{~S}$

\title{
Inhomogeneous magnesium hydride synthesized by low temperature ion implantation: weak localization effect
}

\author{
P. Nédellec $\left({ }^{1}\right)$, L. Dumoulin( $\left.{ }^{1}\right)$, J.P. Burger $\left({ }^{2}\right)$, H. Bernas $\left({ }^{1}\right)$, H. Köstler $\left({ }^{3}\right)$ \\ and $A$. Traverse $\left({ }^{1}\right)$ \\ ( $\left.{ }^{1}\right)$ Centre de Spectrométrie Nucléaire et Spectrométrie de Masse CNRS-IN $\mathrm{P}_{3}, 91405$ \\ Campus Orsay, France \\ $\left(^{2}\right)$ ESPCI Laboratoire de Physique des Solides, 10 rue Vauquelin, 75005 Paris, France \\ $\left({ }^{3}\right)$ Universität Ulm, Sektion Kernresonanzspektroscopie, D-7900 Ulm-Donau, Germany
}

(Received 11 June 1993, accepted in final form 21 July 1993)

Résumé. - Des films d'hydrure $\mathrm{MgH}_{x}$ sont préparés, hors de l'équilibre thermodynamique, par implantation d'ions $\mathrm{H}$ dans des films de $\mathrm{Mg}$ maintenus à $5 \mathrm{~K}$. La mesure de la résistance et de la magnétorésistance en fonction de la température met en évidence des effets importants de localisation électronique due au désordre atomique. Pour les faibles concentrations d'hydrogène, $x \leq 0,3$, la conductivité varie comme $\sigma \sim \log (T)$, variation caractéristique d'un comportement dominé par les effets de localisation électronique faible à 2 dimensions. La résistivité est très sensible aux effets d'inhomogénéité, liés à la diffusion de $\mathrm{H}$. Nous avons modélisé ces effets en introduisant un coefficient géométrique variant avec la température. Pour les concentrations plus élevées $(0,7 \leq x \leq 3)$, après un recuit à $20 \mathrm{~K}, 50 \mathrm{~K}$ et $110 \mathrm{~K}$, les films montrent également des effets de localisation mais la conductivité varie maintenant comme $\sigma \sim \sqrt{T}$, caractéristique d'un comportement tridimensionnel. L'analyse des résultats est compatible avec l'hypothèse d'un système inhomogène formé du mélange de deux phases avec des propriétés électriques contrastées : un "mauvais métal" et un "bon métal" qui, à basse température, pourrait présenter un état supraconducteur non percolant.

\footnotetext{
Abstract . - Metastable $\mathrm{MgH}_{x}$ hydride was prepared by $\mathrm{H}$ ion implantation into $\mathrm{Mg}$ films at $5 \mathrm{~K}$. The resistivity and magnetoresistance temperature dependence reveal weak localization effects due to atomic disorder. At low hydrogen concentrations, $x \leq 0.3$, the conductivity varies as $\sigma \sim \log (T)$, typical of two-dimensional weak localization behaviour. The resistivity is also very sensitive to the sample inhomogeneity, due to $\mathbf{H}$ diffusion, which can be modelled by introducing a temperature-dependent geometrical percolating factor $G$. At higher $\mathrm{H}$ concentrations, $0.7 \leq$ $x \leq 3$, after annealing at $20 \mathrm{~K}, 50 \mathrm{~K}$ and $110 \mathrm{~K}$, the samples also exhibit weak localization but with three-dimensional behaviour i.e. $\sigma \sim \sqrt{T}$. Our analysis is consistent with the existence of an inhomogeneous system formed by a mixture of two phases with contrasted conduction properties, one of which is a well-behaved metal, while the other displays the localization properties. The results lead us to identify the former phase to a non percolating superconducting phase at low temperature.
} 


\section{Introduction.}

In a previous experiment [1], we showed that the preparation of $\mathrm{MgH}_{x}$ hydride by $\mathrm{H}$ ion implantation at low temperature $(5 \mathrm{~K})$ leads to a situation quite different from the usual insertion of hydrogen under pressure at temperatures above $300 \mathrm{~K}$. In the equilibrium-thermodynamics case, the interstitial $\mathrm{H}$ at low $\mathrm{H}$ concentrations occupies the tetrahedral site in the hexagonal structure of $\mathrm{Mg}$, while at higher $\mathrm{H}$ concentrations a hexagonal-tetragonal transition occurs, leading to the formation of a stable insulating dihydride $\mathrm{MgH}_{2}$. Low-temperature proton implantation, even at $\mathrm{H}$ concentrations up to $x \sim 3$, led to no structural change and no insulating state was observed. A disordered $\mathrm{MgH}_{x}$ hydride was found; large low-temperature $(T \leq 110 \mathrm{~K})$ annealing effects showed the instability of this material. This clearly shows that low-temperature implantation-in which kinetic effects are quenched-leads to a phase diagram which differs from equilibrium. However, as we show here, kinetic blocking does not prevent the formation of a two-phase system. We have now made electrical resistivity measurements that allow us to analyze the effect of disorder via the resistivity variation with temperature and magnetic field $B$. Our results show i) weak localization effects ii) inhomogeneity effects and iii) evidence for the existence of a non-percolating superconducting phase at low magnetic field and low temperature.

The weak electronic localization (WEL) theory [2] allows us to analyze the temperature and magnetic field-dependent resistivity for $\mathrm{H}$ concentrations below 0.05 . At higher concentrations we are led to assume the existence of a random biphase system. One of the phases is highly disordered and provides the major contribution to weak localization effects; the other phase is composed of small, weakly connected metallic domains which, as we shall discuss below, may display superconductivity at low temperature. The existence of sample inhomogeneity may be related to quantum diffusion and/or strain-induced diffusion of the implanted hydrogen. The evidence for inhomogeneity will be discussed below, although it is difficult to provide a clear description of the morphology of the inhomogeneities.

In the present paper, after describing (Sect. 2) the preparation and measurement techniques of implanted $\mathrm{MgH}_{x}$ films, we present (Sect. 3) results for i) low hydrogen concentration $(x \leq 0.3)$ analyzed in terms of the WEL effects on the resistivity temperature and magnetic field dependence; ii) annealing effects at different temperatures, i.e., after varying degrees of $\mathrm{H}$ diffusion and defect annealing, and iii) high $\mathrm{H}$ concentrations with $0.7 \leq x \leq 3$. The magnetoconductance and resistivity $\rho(T)$ variations for $x=0.3$ could only be accounted for by assuming the existence of an inhomogeneous sample with WEL effects in one of its components and precursor effects of superconductivity in the other. Annealing leads to a residual resistivity decrease and also to a decrease of the proportion of the superconducting phase, which seems to have essentially identical $T_{\mathrm{c}}$ values in different (isolated) domains i.e. its intrinsic features are nearly independent of the total hydrogen concentration $x$. Section 4 is devoted to a discussion of the possible features of the superconducting state and the reasons for the spatial heterogeneity of $\mathrm{MgH}_{x}$ films prepared by low temperature implantation.

\section{Preparation and characterization.}

Four identical films were prepared by evaporation of pure $\mathrm{Mg}$ on the same quartz substrate from an electron gun source in a vacuum of $10^{-9}$ torr. The average thickness as deduced from the resistivity ratio measurements was $58 \mathrm{~nm}$, in agreement with Rutherford Backscattering measurements. The substrate was held at room temperature during the evaporation and the films were overlayed with $57 \mathrm{~nm}$ of $\mathrm{SiO}$. For low temperature $\mathrm{H}$ implantation, the quartz 
substrate was mounted in a He cryostat coupled to the implanter [3], allowing a complete in situ study of the resistance, temperature and magnetic field dependence of the $\mathrm{Mg}$ films in the range $1.5 \mathrm{~K}<T<110 \mathrm{~K}$ and $0<B<4 \mathrm{~T}$ at each fluence step. The hydrogen implantations were performed at $5 \mathrm{~K}$ and the incident energy of $4 \mathrm{keV}$ was chosen so that the implantation profile was nearly constant in the $\mathrm{Mg}$ film due to the presence of $\mathrm{SiO}$ overlay. A fluence of $3 \times 10^{17} \mathrm{H} / \mathrm{cm}^{2}$ corresponds roughly to a concentration $x=1 \mathrm{H} / \mathrm{Mg}$; fluences up to $9 \times 10^{17} \mathrm{H} / \mathrm{cm}^{2}$ were implanted. Other details on the experimental technique are given in reference [1].

The electrical resistance and the magnetoresistance, i.e. $R(T, B)$, were measured using a low-frequency high-resolution ac bridge and a four-point probe technique. In order to prevent heating and subsequent film modifications, the ac current was maintained below $500 \mathrm{nA}$.

The initial resistance changed with time even at low temperature, an effect which was larger at larger concentrations and whose exact meaning is not entirely understood. In order to measure the transport properties of stable films, we annealed them at $20 \mathrm{~K}, 50 \mathrm{~K}$ and $110 \mathrm{~K}$. Annealing at $20 \mathrm{~K}$ tends to stabilize the overall inhomogeneity of the $\mathrm{MgH}_{x}$, only weakly changing the physical properties of the films. In contrast, annealing at $50 \mathrm{~K}$ and $110 \mathrm{~K}$ strongly affected the residual resistivity of the films. These points will be analyzed in the next section.

\section{Results and analysis.}

3.1 LOW HYDROGEN CONCENTRATION SYSTEMS $(x \leq 0.3)$. - The first important feature concerns the very large resistivity increase with hydrogen concentration for $x \leq 0.3$ : the slope $\frac{\mathrm{d} \rho}{\mathrm{d} x}$ is equal to $2 \mu \Omega . \mathrm{cm}$ per at. \%H. This slope is systematically lower for $x \geq 0.7$.

Figure 1 shows the resistivity temperature-dependence $\rho(T)$ in zero magnetic field for a pure $\mathrm{Mg}$ film and for the same film charged with $30 \%$ hydrogen. The pure $\mathrm{Mg}$ film (Fig. 1a) definitely displays metallic behaviour associated with a resistive ratio $\frac{\rho(290 \mathrm{~K})}{\rho(4.2 \mathrm{~K})}=6$. The $\mathrm{H}$-implanted film shows a negative temperature coefficient of the resistivity (TCR) at low temperature, and the TCR is larger after annealing at $50 \mathrm{~K}$ (Fig. 1b) than after a $110 \mathrm{~K}$ anneal (Fig. 1c). The hydrogen implantation has enhanced disorder, leading to the WEL regime, which is well understood both experimentally and theoretically [2]. Annealing the same film at higher temperatures leads to a reduction in the degree of disorder, i.e., the residual resistivity decreases. Simultaneously the resistivity minimum which characterizes the competition between the phonon contribution and localization effects shifts towards lower temperatures ( $16 \mathrm{~K}$ after a $50 \mathrm{~K}$-anneal and $10 \mathrm{~K}$ after annealing at $110 \mathrm{~K}$ ).

The localization effect is also clearly recognized in figure 2 , which shows the magnetoconductance $\Delta C_{\square}(B)=C_{\square}(B)-C_{\square}(0)$ at $1.5 \mathrm{~K}$ for the pure $\mathrm{Mg}$ and $\mathrm{MgH}_{x}(x=0.05$ and $x=0.15)$ samples. $C_{\square}$ is the conductance per square which is the relevant parameter in the two-dimensional (2D) WEL régime. In the low field limit, the pure $\mathrm{Mg}$ film $\left(R_{\square}=0.17 \Omega / \square\right)$, as shown in figure 2a, follows Kohler's law with a parabolic conductivity dependence and a negative magnetoconductance: $\Delta C_{\square} \sim-B^{2}$ (solid line in Fig. 2a). As the magnetic field increases (for $B>1 \mathrm{~T}$ ), we expect the perturbation of electron motion due to the magnetic field to overcome the effect of the crystal lattice periodic potential so that magnetic breakdown occurs [4]: these effects change the magnetoconductance variation but not its sign. For the $x=0.05$ film (corresponding to $R_{\square}=2.6 \Omega / \square$ ) a sign inversion of the magnetoconductance occurs at low field. Indeed, the magnetoconductance is presumably due to two contributions: 


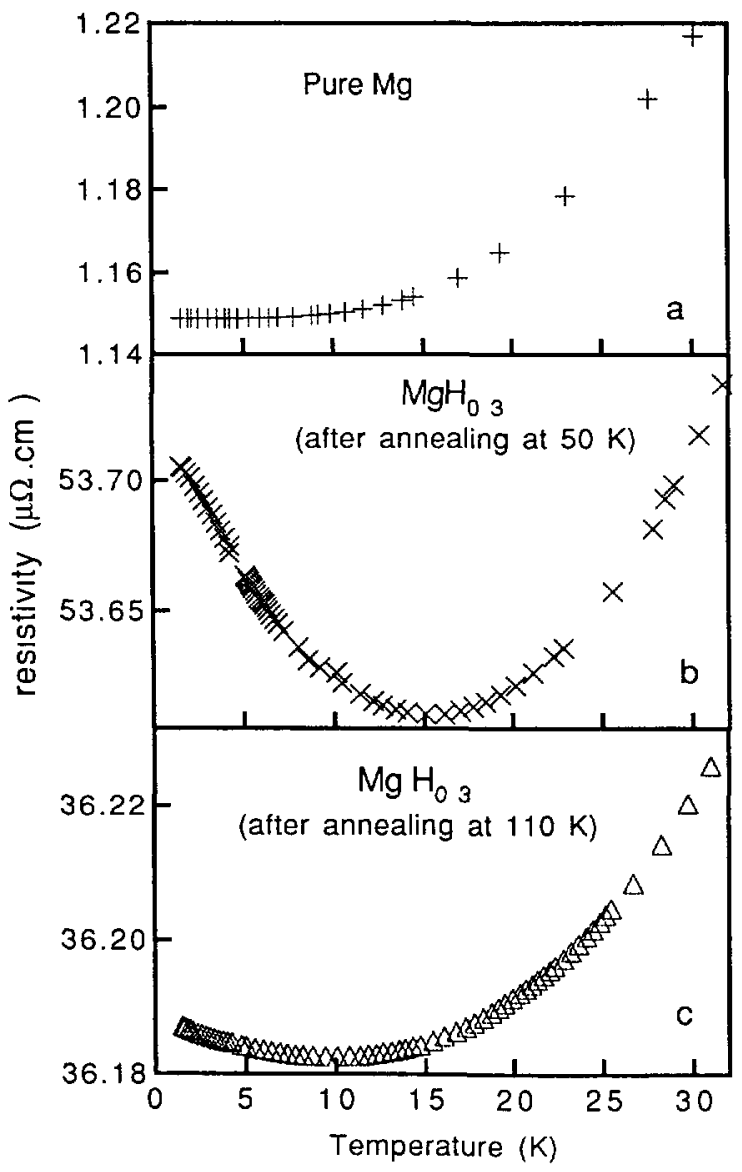

Fig. 1. - Temperature variation of the resistivity for a pure $\mathrm{Mg}$ film and the same film charged with $\mathrm{H}$ annealed at first at $50 \mathrm{~K}(\mathrm{~b})$ then at $100 \mathrm{~K}(\mathrm{c})$.

the negative normal magnetoconductance given by Kohler's rule whose amplitude decreases as the inverse square of $\rho$, and the anomalous positive magnetoconductance due to localization, since $\mathrm{MgH}_{x}$ is certainly a weak spin-orbit coupling conductor. The solid line in figure $2 \mathrm{~b}$ is a theoretical fit obtained by describing the WEL contribution by [4]:

$$
\Delta C_{\square}(B)=\frac{e^{2}}{2 \pi^{2} \hbar}\left[\log \left(\frac{B}{B_{\varepsilon}}\right)+\Psi\left(\frac{1}{2}+\frac{B_{\varepsilon}}{B}\right)\right]
$$

$B_{\varepsilon}$ is the characteristic inelastic temperature dependent field connected to the inelastic coherence length $L_{\varepsilon}$ by the relation:

$$
L_{\varepsilon}=\sqrt{\frac{\hbar}{4 e B_{\varepsilon}}}
$$

Equation (1) holds in the presence of a perpendicular magnetic field $B$ and for 2D systems, i.e., when the condition $d$ (film thickness) $<L_{\varepsilon}$ is verified: this is our case as $L_{\varepsilon}=140 \mathrm{~nm}$ at $1.4 \mathrm{~K}$. 


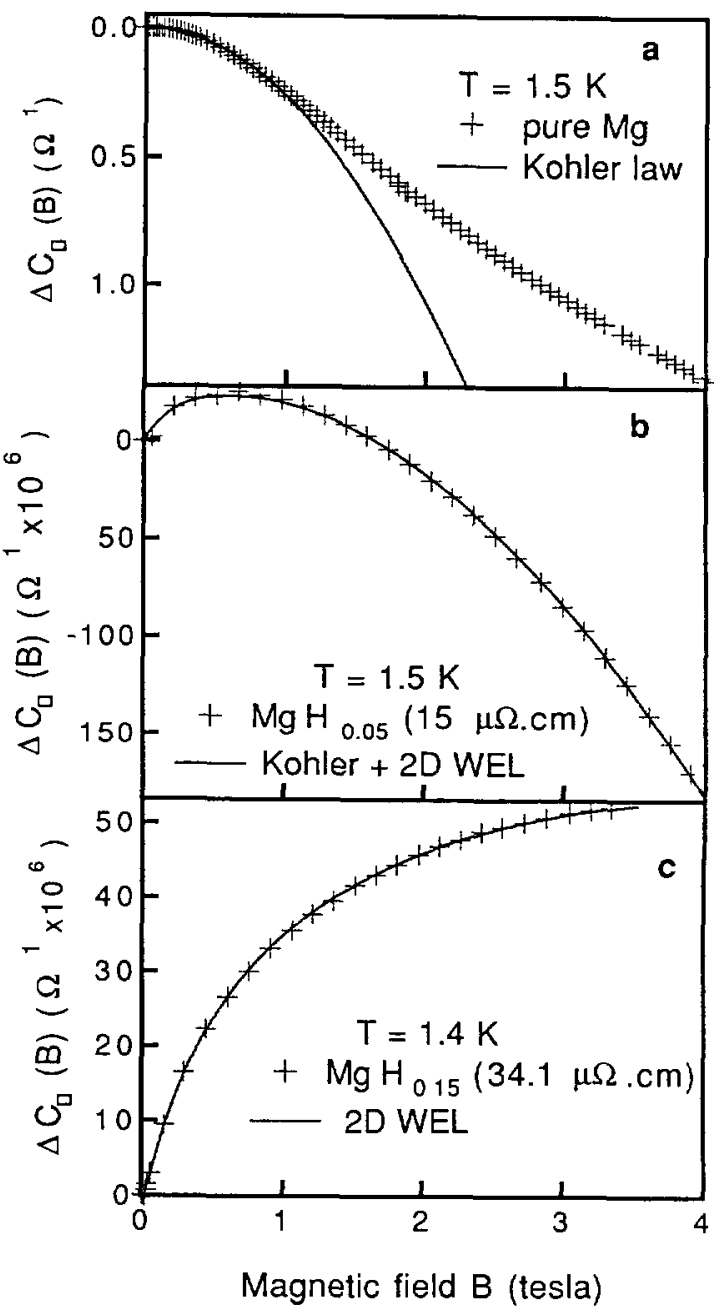

Fig. 2. - Magnetoconductance $\Delta C_{\square}(B)=C_{\square}(B)-C_{\square}(0)$ at $1.5 \mathrm{~K}$ for a film of (a) pure $\mathrm{Mg}$, (b) $\mathrm{MgH}_{0.05}$ and (c) $\mathrm{MgH}_{015}$. The solid lines correspond to the theoretical variations of the classical and weak electron localization contributions.

As the $\mathrm{H}$ concentration is increased (Fig. 2c), the positive contribution (weak localization) to the magnetoconductance dominates as the Kohler contribution is weakened due to electron mobility decrease. Although the shape of the experimental curve is consistent with equation (1) the absolute $\Delta C_{\square}(B)$ values must be adjusted by a factor ranging from 1 to 1.63 for $x=0.15$. This has led us to modify equation (1) which is only valid for a homogeneous system, by introducing a factor $G$ :

$$
\Delta C_{\square}(B)=\frac{e^{2}}{2 \pi^{2} \hbar} G\left[\log \left(\frac{B}{B_{\varepsilon}}\right)+\Psi\left(\frac{1}{2}+\frac{B_{\varepsilon}}{B}\right)\right]
$$

The physical interpretation of this factor $G$ is the existence of percolation in our system. Consider a mixture of two phases 1 and 2 in two extreme situations. First, consider the case 
in which phase 2 is an insulator (for instance holes or cracks in a metallic film) characterized by a volume fraction $p$ and a conductivity $\sigma_{2}=0$; phase 1 is the conducting part with a conductivity $\sigma_{1}$ at the crystallite scale. At low $p$ values, the mixture is a conductor and changes to an insulator at the percolation threshold $p_{\mathrm{c}}$. The conductivity variation reflects the percolation law $\sigma=\sigma_{1}\left(p_{\mathrm{c}}-p\right)^{t}$ where $t \sim 1.3$ for a $2 \mathrm{D}$ system. $G$ is related here to the term $\left(p_{\mathrm{c}}-p\right)^{t}$ and is a measure of the "effective width" (assuming two conductors in parallel) of the remaining conducting phase. As the sample approaches the percolating threshold $p_{\mathrm{c}}$ from below, a reduction of $G$ from 1 for the homogeneous samples down to zero is observed [6-9]. For our samples, the analysis indicates an inverse variation, namely an increase of $G$ as the hydrogen concentration increases. This behaviour is suggestive of another extreme case, in which phase 1 is a superconductor $\left(\sigma_{1}=\infty\right)$ and phase 2 is a normal metal with a volume fraction $p$. The conductivity divergence is then described by the percolation law: $\sigma \sim \sigma_{2}\left(p-p_{\mathrm{c}}\right)^{s}$ with $s \sim t$ for $2 \mathrm{D}$ systems. As $p$ approaches $p_{\mathrm{c}}$ from above, $\sigma$ and $G$ increase as observed in our case.

This percolation picture corresponds to well-contrasted phases, while our $\mathrm{MgH}_{x}$ films are probably in fact a more intimate mixture of two different materials, both with finite conductivities $\sigma_{1}$ and $\sigma_{2}$. Nevertheless, our results show that inside phase 1 there must be a small fraction of isolated domains which become superconducting at low $T$ and which are in contact with normal metal domains, while phase 2 is a normal disordered metal displaying Anderson localization. In order to analyze our experimental results we retain the percolation model by using a scaling argument [10]: the conductivity of a binary mixture near the threshold $p_{\mathrm{c}}$ depends on the conductivities of both constituents $\sigma_{1}$ and $\sigma_{2}\left(\sigma_{1}>\sigma_{2}\right)$ and is given by:

$$
\sigma=\sigma_{1}^{\frac{s}{s+t}} \sigma_{2}^{\frac{t}{s+t}}
$$

This relation reduces to $\sigma \sim \sqrt{\sigma_{1} \sigma_{2}}$ in the $2 \mathrm{D}$ limit for which $s \sim t$. It allows us to estimate the factor $G$. Note that effective medium theories, whose relevant parameters are also the volume fractions and conductivities of the various phases, give the same result [11]. By assuming that weak localization takes place only in the less metallic medium 2 , the correction to the conductivity is:

$$
\Delta \sigma=G \Delta \sigma_{2} \quad \text { and } \quad G \sim \sqrt{\frac{\sigma_{1}}{\sigma_{2}}}
$$

The factor $G$ renormalizes the weak localization contributions. We may deduce it from the magnetoconductance and apply the resulting values to the measured conductance in order to check consistency.

Let us now consider the magnetoconductance temperature variation for the sample with $x=0.3$ : the data are fitted using equation (3) (Fig. 3). The fit is excellent with this modified 2D weak localization theory and we have extracted the parameters $G$ and $B_{\varepsilon}$ for different temperatures. $B_{\varepsilon}$ follows a $T^{2}$-law for temperatures larger than $4 \mathrm{~K}$; this variation is consistent with an electron-phonon scattering mechanism [12], the predominant process at high temperatures. Electron-electron scattering can also give the same temperature dependence at lower temperatures [13]. Below $3 \mathrm{~K}$ we observe an unusual increase of $B_{\varepsilon}$ with decreasing temperature: this result is counter to expectations in a homogeneous sample, since the inelastic scattering probability should decrease with $T$. We surmise that it is due to the failure of equation (3) to approximate WEL in the heterogeneous structure at very low temperature. Figure 4 shows the conductance variation with temperature for the same sample $(x=0.3)$. It is known that if the magnetoconductance variation is due to $2 \mathrm{D}$ weak localization, the expected conductance-temperature variation should follow a logarithmic law; this is not observed in our case since the conductance saturates at low temperature. 


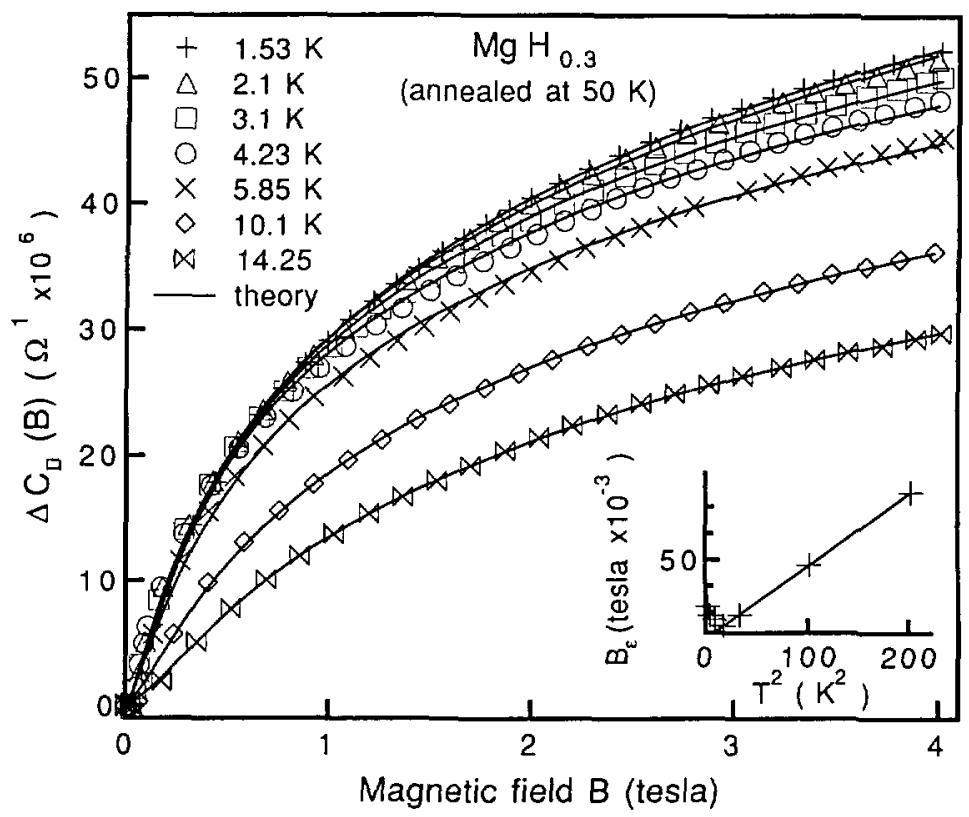

Fig. 3. - Positive magnetoconductance of a $\mathrm{MgH}_{0.3}$ film at different temperatures. The solid lines are the fits to equation (3). The deduced inelastic magnetic field $B_{\varepsilon}$ are shown in the inset.

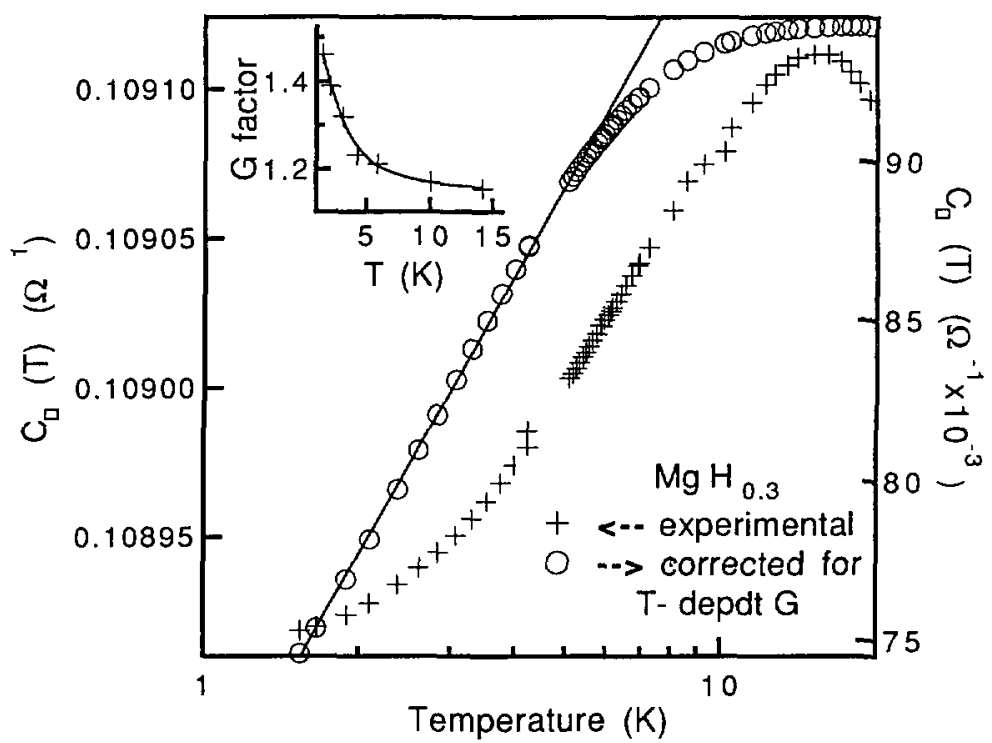

Fig. 4. - The experimental variation ( + ) of the conductance can be transformed into a 2D logarithmic variation (o) with temperature by taking into account the coefficient $G$ related to the inhomogeneity. $G$ is deduced from the magnetoconductance analysis. 
The magnetoconductance results are consistent with a 2D WEL contribution, modified by the temperature dependent coefficient $G: G$ increases when the temperature is lowered (see inset, Fig. 4). If we correct, in the same way, the experimental $\Delta C_{\square}(T)$ variation with the $G$ factor deduced from the magnetoconductance analysis, we obtain the expected logarithmic variation. From equation (5) the $G$ temperature-dependence can be related to an increase of $\sigma_{1}$. Such a variation of the conductivity of the more metallic part of the sample would occur if it neared superconductivity in this temperature range. This interpretation is clearly speculative at this point, but we have found no other explanation in the framework of the simple approach described by equations (3) and (5) which fit the data remarkably well.

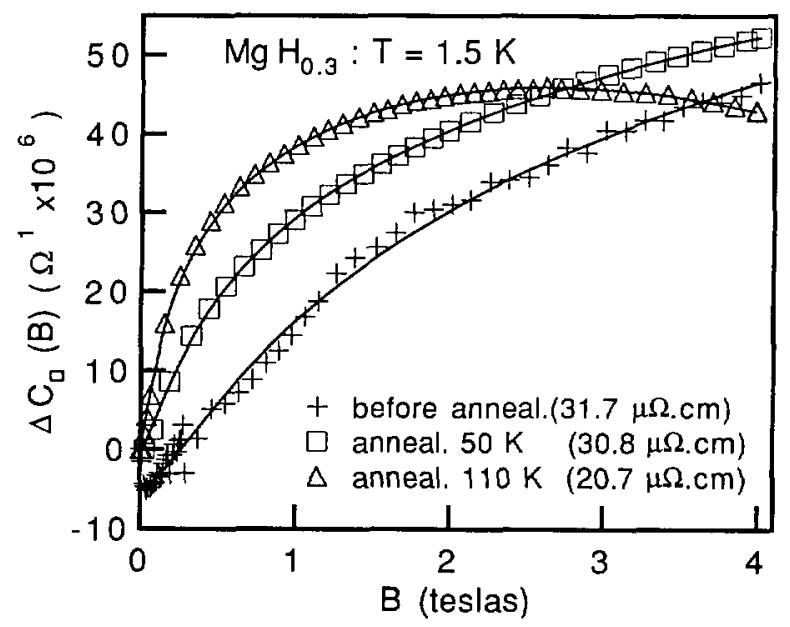

Fig. 5. - Magnetoconductance curves of an $\mathrm{MgH}_{0.3}$ film for different annealing temperatures. The solid lines are calculated with the weak localization and Kohler contributions. Note the negative magnetoconductance for the non annealed film.

3.2 ANNEALING EFFECTS. - In order to further understand the role of hydrogen in our sample, we have analyzed the annealing effect on magnetoconductance. Representative curves of the magnetoconductance variation are shown in figure 5 for different annealing temperatures. Raising the annealing temperature decreases the resistivity, and the decrease is enhanced above $50 \mathrm{~K}$. The positive part of the magnetoconductance is still ascribable to $2 \mathrm{D}$ WEL as in equation (3), but there are two deviations: i) the less resistive sample shows a magnetoconductance maximum, clearly due to the Kohler negative magnetoconductance contribution; ii) different magnetoconductance signs are observed for the non-annealed film in the low field region: the negative part decreases rapidly as the field increases. Without swelling on this effect in detail, we note that the negative part disappears in the same way as the superconducting state vanishes with applied magnetic field. In order to compare quantitatively with equation (3), we treat it as a constant contribution. The factor $G$ decreases with increasing annealing temperature from $G=2.3$ to 1.46 ( $50 \mathrm{~K}$ annealing) and 1.17 (110 $\mathrm{K}$ annealing). According to equation (5), this decrease can be related to an increase of the conductivity of the less resistive phase as hydrogen moves inside the sample even at low temperatures. This is consistent with our 
interpretation of $G$, and is related to the difference in heterogeneity between the local and the macroscopic scales.

We wish to stress that, whether or not superconductivity occurs in the particular material, our analysis shows how to obtain a signature of the existence of the WEL in an inhomogeneous sample.

3.3 High HYDROGEN CONCENTRATION $(0.5 \leq x \leq 3)$. - In reference [1] we found a marked decrease in the electrical resistivity concentration dependence $\frac{\mathrm{d} \rho}{\mathrm{d} x}$ at higher concentrations. For $x>0.7$, the slope $\frac{\mathrm{d} \rho}{\mathrm{d} x} \sim 0.3 \mu \Omega . \mathrm{cm}$ per at. $\% \mathrm{H}$ is about one order of magnitude smaller than at concentrations $x \leq 0.3$ for which $\frac{\mathrm{d} \rho}{\mathrm{d} x} \sim 2 \mu \Omega$.cm per at. \% $\mathrm{H}$. In these high-concentration samples annealing reduces atomic disorder as well as the proportion of superconducting phase. The superconducting properties are most apparent around $x \sim 0.7$ and do not vary for larger concentrations. This indicates that the superconducting phase corresponds to a nearly constant $x$ value. At higher concentrations, excess hydrogen presumably mostly travels to grain boundaries.

Figure 6 shows the resistivity temperature dependence after annealing at $110 \mathrm{~K}$. At this temperature the $\mathrm{H}$ atoms certainly diffuse very quickly. The temperature below which the resistivity increases with decreasing temperature becomes higher with increasing $\mathrm{H}$ concentration. For $x \sim 3$ no resistivity minimum is observed below $100 \mathrm{~K}$. The resistivity temperature dependence below the minimum is close to the $\sqrt{T}$ law characterizing the three dimensional (3D) weakly régime. At temperatures below $10 \mathrm{~K}$, the conductivity is always proportional to $\sqrt{T}$ for concentrations $x>0.7$ as seen in figure 7 : this temperature dependence is entirely characteristic of 3D localized samples. This also correlates well with the magnetoconductance variation which is always positive, i.e., characteristic of a weak localization régime.

However the conductivity slope $\frac{\mathrm{d} \sigma}{\mathrm{d} \sqrt{T}}$ does not have a constant value, independent of the disorder, as predicted in the weak localization régime. This anomaly has several consequences: i) it modifies the decrease of the residual resistivity - the slope $\frac{\mathrm{d} \sigma}{\mathrm{d} \sqrt{T}}$ is roughly proportional to the resistivity; ii) at lower temperature we also observe (Fig. 7) small deviations from the $\sqrt{T}$ law below $2 \mathrm{~K}$ for all concentration $x \geq 0.7$, corresponding to a conductivity increase. This deviation is more pronounced for films before annealing and it disappears for films annealed at $110 \mathrm{~K}$. This conductivity increase at low temperature can be related to a very dilute, unstable superconducting phase; iii) the ratios between the slopes $\frac{\mathrm{d} \sigma}{\mathrm{d} \sqrt{T}}$ for different $\mathrm{H}$ concentrations are not modified by annealing.

Figure 8 shows typical magnetoconductance curves at different temperatures in a perpendicular magnetic field. The observed positive magnetoconductance in our $\mathrm{MgH}_{x}$ films with $x \geq 0.7$ reproduces the main features of the expected theoretical variation [14] and indicates the presence of $3 \mathrm{D}$ localization, confirming the one deduced from the conductivity temperature dependence. At low temperature we observe an anomalous negative magnetoconductance which increases with decreasing temperature: this anomaly is observed only at low magnetic fields and disappears for $B \geq 0.1 \mathrm{~T}$ independently of the $\mathrm{H}$ concentration; this can also be related to the assumed superconducting phase. For high-temperature anneals, this magnetoconductance is also weakened. We have assumed the existence of two contributions to the magnetoconductance: one is an offset which accounts for the negative (superconducting ?) contribution, the other is the positive 3D WEL magnetoconductance (14] (the function $F_{3}(x)$ 


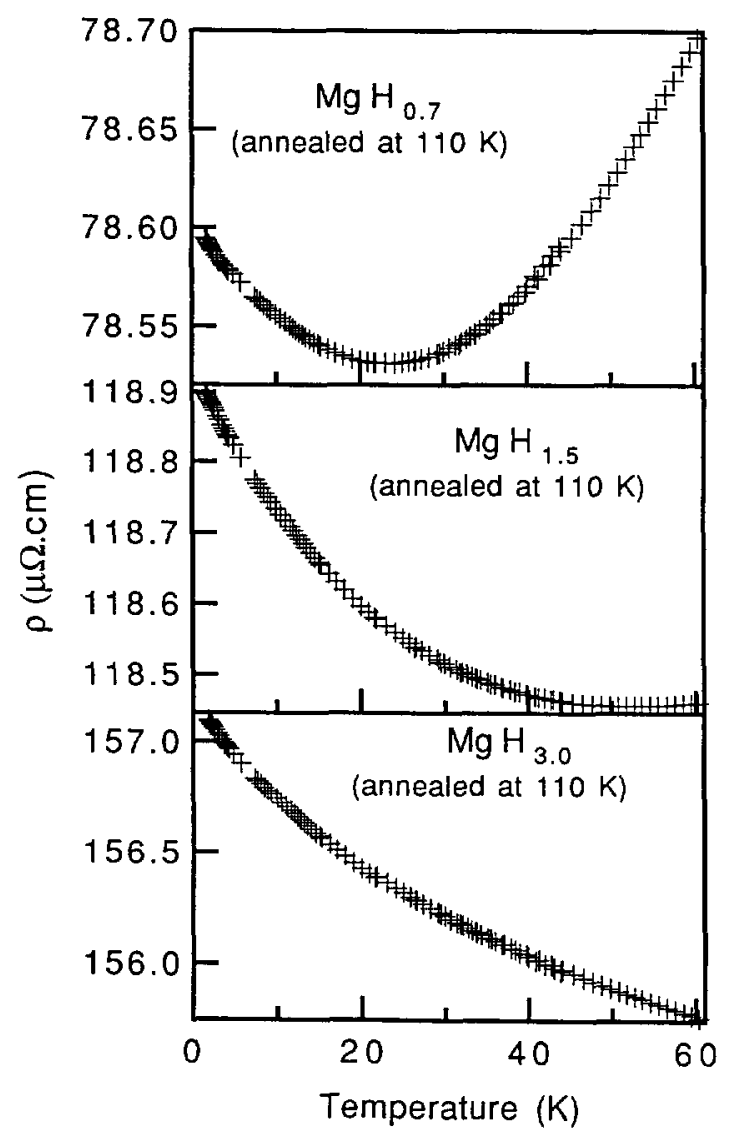

Fig. 6. - Resistivity temperature dependence of the high concentration films annealed at $110 \mathrm{~K}(x=$ $0.7,1.5$ and 3$)$.

is defined in Ref. [14]):

$$
\Delta \sigma(B)=\text { offset }+\frac{e^{2}}{2 \pi^{2} \hbar} G \sqrt{\frac{e B}{\hbar}} F_{3}\left(\frac{B}{B_{\varepsilon}}\right)
$$

The fitting is performed with the parameter $B_{\varepsilon}$, and with $G$ which describes the sample's inhomogeneity. The agreement obtained for the positive magnetoconductances (Fig. 8) is remarkable. The factor $G$ is again temperature-dependent and decreases with increasing temperature: the result is similar to the analysis of the $\mathrm{MgH}_{0.3}$ films. The determination of $B_{\varepsilon}$ gives a diffusion length $L_{\varepsilon}$ around $50 \mathrm{~nm}$ which is somewhat shorter than the film thickness, i.e., it favours the $3 \mathrm{D}$ situation, but the difficulties met in the low $\mathrm{H}$ concentration limit (Fig. 3) occur again insofar as the interpretation of $B_{\varepsilon}$ is concerned (see the non-monotonic temperature dependence of $B_{\varepsilon}$ ).

\section{Discussion.}

Hydrogen insertion in $\mathrm{Mg}$ by low-temperature implantation leads to a new hydride. We have 


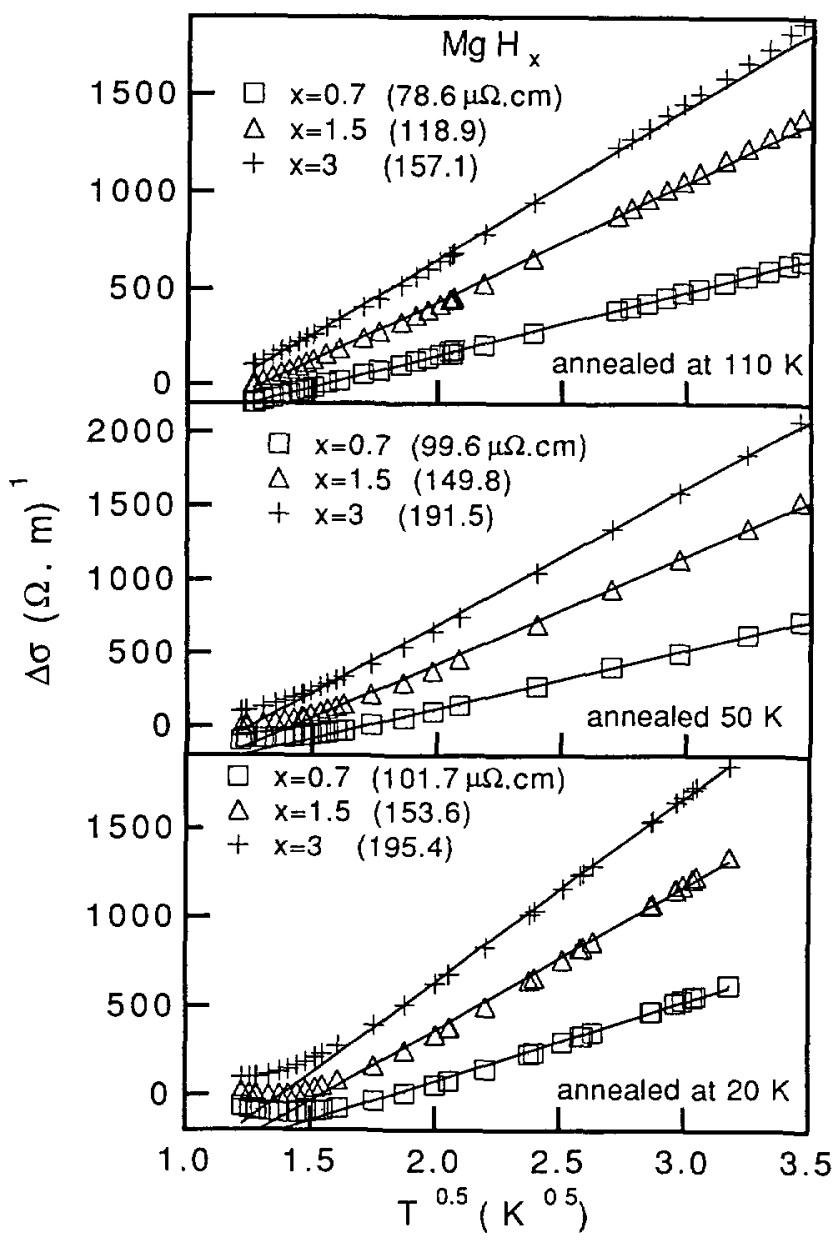

Fig. 7. - Conductivity temperature dependence for three different films ( $x=0.7,1.5$ and 3$)$ annealed at $20 \mathrm{~K}, 50 \mathrm{~K}$ and $110 \mathrm{~K}$. For the first two anneals, the conductivity begins to increase at $T \leq 2 \mathrm{~K}$. This is related to the non-percolating superconducting phase discussed in the text. (The curves have been shifted vertically).

observed and analyzed several different effects:

1) due to atomic disorder, WEL affects the resistivity. This is well established experimentally by the resistance and magnetoresistance temperature variation. The WEL is governed, in large part, by the hydrogen concentration as we go from the $2 \mathrm{D}$ limit at low $\mathrm{H}$ concentration to $3 \mathrm{D}$ at high $\mathrm{H}$ concentration $(x>1.5)$. For $x=3$ the resistivity minimum occurs beyond $100 \mathrm{~K}$, a temperature which is much higher than that found for other metals with similar localization effects. The increase of the resistivity below $100 \mathrm{~K}$ cannot be related to semiconducting behaviour because $\rho(T)$ is proportional to $\sqrt{T}$ with no exponential law.

2) From our analysis, there is evidence of a more complicated effect related to the inhomogeneity of $\mathrm{MgH}_{x}$. we are led to postulate the existence of more than one pure homogeneous disordered phase. These different phases are likely both disordered, with different $\mathrm{H}$ concentrations and hence different electronic structures. But due to low-temperature diffusion of 


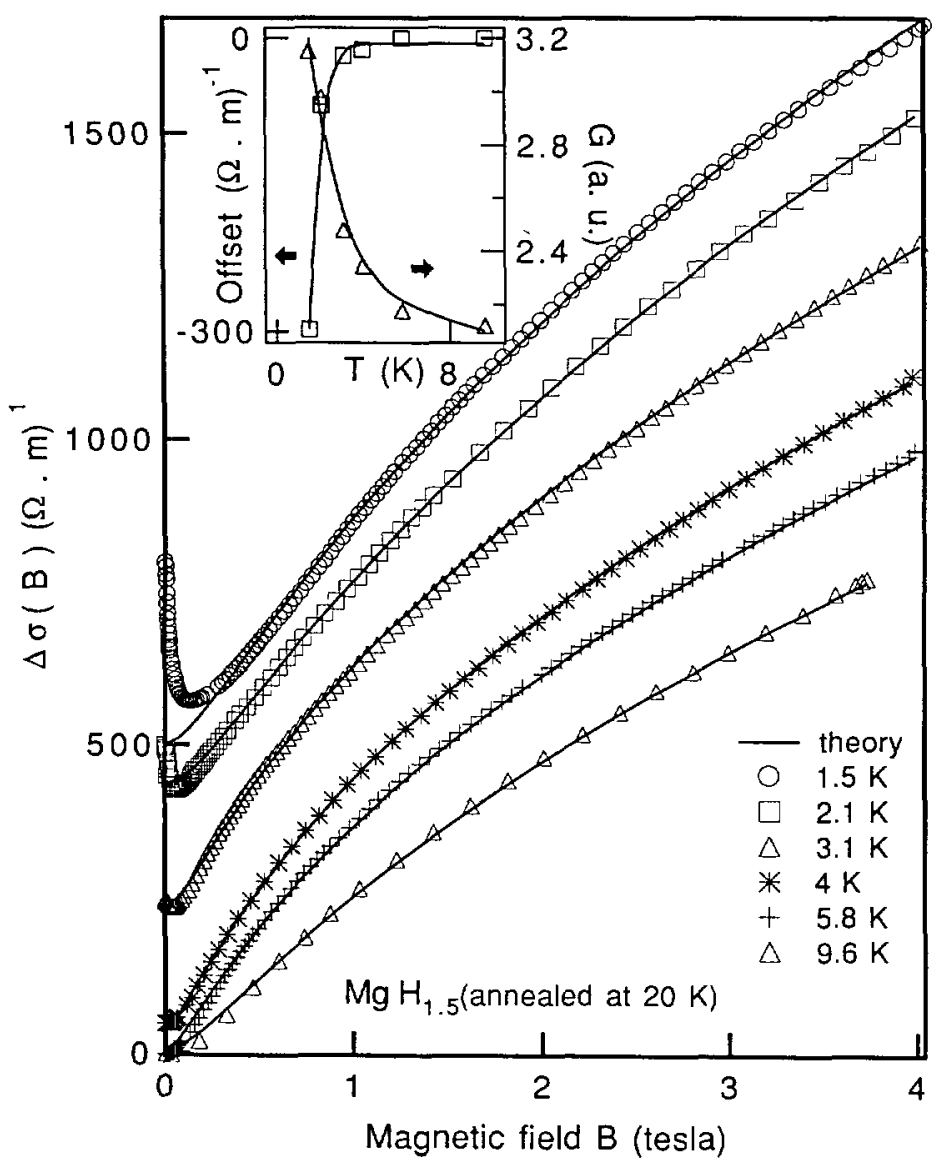

Fig. 8. - Negative and positive magnetoconductance for $\mathrm{MgH}_{1.5}$ at different temperatures. For clarity the curves have been offset relative to each other. The vertical scale corresponds to the lowest curve. The solid lines correspond to the positive 3D weak electron localization (Eq. (6)) and the resulting values for $G$ and the offset are shown in the inset.

hydrogen and the instability of these different phases there are annealing effects at low temperature which decrease the atomic disordered and hence the residual resistivity, as well as the resistivity temperature minimum due to localization effects. An important feature is the percolation law due to this inhomogeneity, which was analyzed for $x=0.3$ and $x=1.5 \mathrm{using}$ the magnetoconductance results.

3) Our results suggest the existence of a superconducting phase in $\mathrm{MgH}_{x}$. The experimental evidence for this is i) the small increase of $\Delta \sigma$ (Fig. 7 ) below $T=1.5 \mathrm{~K}$; ii) the existence, at low magnetic fields, of a negative magnetoconductance (Fig. 8) related to the superconducting critical fields; iii) the increase of the factor $G$ at low temperature which can be explained (Eq. (5)) by the conductivity increase of phase 1 . However, the total resistance never goes to zero which implies that the superconducting phase 1 is very dilute and that the system is well above the percolation threshold. Note that proximity effect-induced superconductivity in normal regions of the sample would also lead to a decrease of $\rho$ at lower temperature.

The existence of a superconducting phase would be in line with existing results on implanted 
hydrides. Low temperature implantation, with kinetic blocking of $\mathrm{H}$ motion, may favour sites in which the electron added by $\mathrm{H}$ contributes to the density at the Fermi energy on the $\mathrm{H}$ site; the formation of $\mathrm{MgH}$ or $\mathrm{MgH}_{1+x}$ could then give $T_{\mathrm{c}}$ values similar to those of $\mathrm{Al}$ or low temperature-implanted $\mathrm{AlH}_{x}$ [15] if the optical phonon energy is sufficiently low. Of course, the transfer of a large fraction of the hydrogen electrons increases the free energy of the metal and does not favour thermodynamic stability [16]. This ties in with the time-dependent conductivity relaxation at $4.2 \mathrm{~K}$ [1]. Along the same lines, annealing at higher temperature can decrease or even erase the superconducting volume fraction, due to higher $\mathrm{H}$ diffusion under elastic stress (Gorsky effect).

\section{Acknowledgements.}

We acknowledge the assistance of $O$. Kaïtasov with the implantations.

\section{References}

[1] Köstler H., Traverse A., Nédellec P., Dumoulin L., Ruault M.O., Schlapbach L., Burger J.P. and Bernas H., J. Phys. F (Cond. Mat.) 3 (1991) 8767.

[2] Lee P.A. and Ramakrishnan T.V., Rev. Mod. Phys. 57 (1985) 287;

Ramakrishnan T.V., Chance and Matter, J. Souletie, J. Vannimenus and R. Stora Eds. (North Holland, Amsterdam, 1987) p.213. and references therein.

[3] Traverse A., Paumier E., Nédellec P., Bernas H., Dumoulin L. and Chaumont J., Phys. Rev. B37 (1988) 2495.

[4] Falicov L.M., Pippard A.B. and Sievert P.R., Phys. Rev. 151 (1966) 498.

[5] Hikami S., Larkin A.I. and Nagaoka Y., Progr. Theor. Phys. 63 (1980) 707.

[6] Van den Dries L., Van Haesendonck C., Bruynseraede Y. and Deutscher G., Phys. Rev. Lett. 46 (1981) 565.

[7] Fehr Y., May-tal S. and Rosenbaum R., Phys. Rev. B33 (1986) 6631.

[8] Palevskii A. and Deutscher G., Phys. Rev. B34 (1986) 431.

[9] Papandreou N. and Nédellec P., J. Phys. I France 2 (1992) 707.

[10] Straley J.P., Phys. Rev. B15 (1977) 5733.

[11] McLachlan D.S., J. Phys. C20 (1987) 865.

[12] Lawrence W.E. and Meador A.B., Phys. Rev. B18 (1978) 1154.

[13] Bergmann G., Phys. Rep. 107 (1984) 1.

[14] Kawabata A., Solid State Commun 34 (1980) 431; J. Phys. Soc. Jpn 49 (1980) 628.

[15] Dumoulin L., Nédellec P., Chaumont J., Gilbon D., Lamoise A.M. and Bernas H., C.R. Acad. Sc. (Paris) 283 (1976) B285.

[16] Griessen R. and Driessen A., Phys. Rev. B30 (1984) 1. 